\title{
3D Membrane Imaging and Porosity Visualization
}

\author{
Ganesh Sundaramoorthi ${ }^{\mathrm{a}}$, Markus Hadwiger ${ }^{\mathrm{a}}$, Mohamed Ben-Romdhane ${ }^{\mathrm{a}}$, Ali R. Behzad ${ }^{\mathrm{b}}$, \\ Poornima Madhavan ${ }^{\mathrm{c}}$ and S. P. Nunes ${ }^{\mathrm{c}^{*}}$
}

${ }^{\mathrm{a} C o m p u t e r, ~ E l e c t r i c a l ~ a n d ~ M a t h e m a t i c a l ~ S c i e n c e s ~ D i v i s i o n ~(C E M S E), ~}$ Visual Computing Center (VCC),

bImaging and Characterization Core Lab, ${ }^{\mathrm{c}}$ Biological and Environmental Science and Engineering Division (BESE), King Abdullah University of Science and Technology (KAUST), 23955-6900 Thuwal, Saudi Arabia

*Corresponding author: suzana.nunes@kaust.edu.sa 


\begin{abstract}
Ultrafiltration asymmetric porous membranes were imaged by two microscopy methods, which allow 3D reconstruction: Focused Ion Beam and Serial Block Face Scanning Electron Microscopy. A new algorithm was proposed to evaluate porosity and average pore size in different layers orthogonal and parallel to the membrane surface. The 3D-reconstruction enabled additionally the visualization of pore interconnectivity in different parts of the membrane. The method was demonstrated for a block copolymer porous membrane and can be extended to other membranes with application in ultrafiltration, supports for forward osmosis, etc, offering a complete view of the transport paths in the membrane.
\end{abstract}

Keywords: segmentation, visualization, porosity characterization, asymmetric membranes, focused ion beam, serial block face microscopy 


\section{Introduction}

Synthetic membranes are integrated in a variety of processes, which go from hemodialysis to water desalination, beverages and dairy product manufacture, gas separation in petrochemical and pharmaceutical industry. They have different requirements of stability, flux and selectivity. Integral asymmetric or multilayer asymmetric porous membranes are used in almost all membrane-based process ${ }^{1-4}$. While the top layer determines the membrane selectivity and the separation capability, the porous sub-layer provides the needed mechanical stability and should not negatively interfere with the transport. Porosity control is relevant for many other reasons. The main drawback of forward osmosis is concentration polarization, which is highly affected by tortuosity and porosity of the substrate layer ${ }^{3,5-7}$. Porosity influences how fast water transfer through membrane and condensation takes place 8. The porosity of porous electrodes and gas diffusion layers in membrane-electrodeassemblies for fuel cell contributes to the efficiency of the reactive conversion ${ }^{9,10}$.

The preparation of asymmetric porous membranes based on block copolymer selfassembly combined to phase inversion, block sacrifice by selective etching or reactive phase separation ${ }^{11-16}$, leads to the formation of a selective top layer with isoporous structure on the top of the membrane and a sub-layer with gradient porosity. Morphology control in solution and in the final asymmetric membrane is decisive for the final membrane application in ultrafiltration.

Well-known methods for pore size characterization are extensively used for membranes

17. Examples are scanning electron microscopy (SEM), atomic force microscopy (AFM), bubble pressure and gas transport, mercury porosimetry, permporometry, BET adsorption 
and thermoporometry. BET gives us quantified information of total surface area or the overall porosity, accessible to the probing gas. SEM and AFM are restricted in most cases to image the membrane surface morphology. 2D imaging of membrane cross-sections is possible by SEM. Porosimetry gives information on average pore size and distribution. However in none of these methods it is possible to have a detailed characterization of porosity in all different layers of the membrane.

As far as morphological characterization is concerned, advanced microscopy and scattering methods have been very useful for membrane characterization ${ }^{11-13}$, including field emission electron microscopy (FESEM) and transmission electron microscopy (TEM) tomography, associated to synchrotron scattering methods. Cryo-TEM and cryo-SEM helped in investigating copolymer assembly in solution ${ }^{13,17-19}$, before the membrane manufacture. We used for the first time cryo-SEM and environmental SEM (ESEM) to image the effect of $\mathrm{pH}$ on stimuli-responsive porous membranes ${ }^{12}$. Mottern et al. ${ }^{20}$ used focus ion beam (FIB) to prepare ceramic membrane samples as single slices for 2D TEM imaging. Kyotani et al. ${ }^{21}$ reported a similar method combined to grazing incidence X-ray diffraction analysis of tubular zeolites.

More recently new methods of tomography or 3D microscopy image reconstruction have become available with potential use in membrane technology, offering then the perspective of having a complete overview of porosity in the whole volume.

Figure 1 summarizes the resolution of different 3D imaging methods and the sampling volume. 


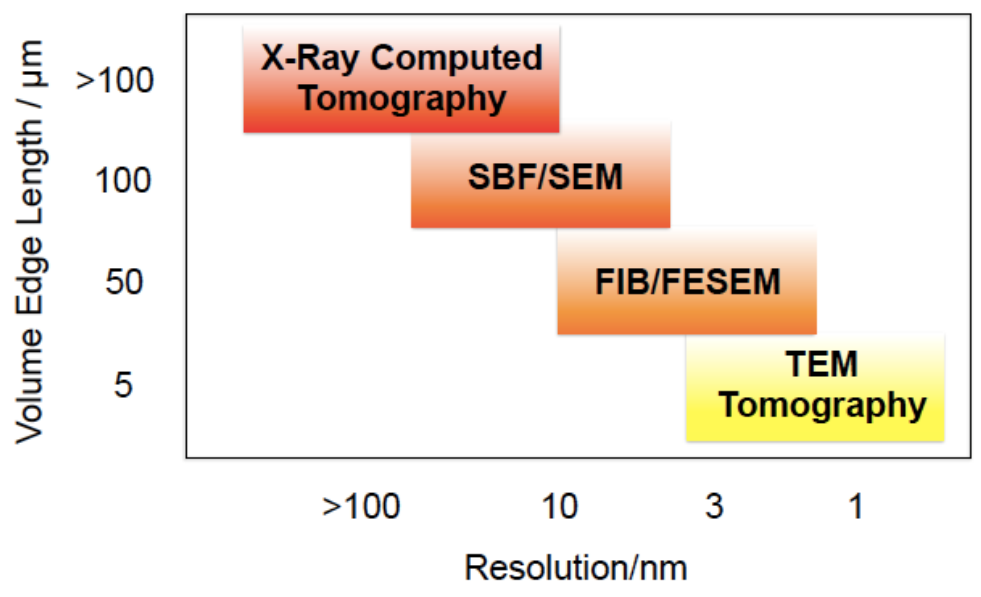

\section{Microfiltration Ultrafiltration Nanofiltration}

Figure 1. 3D Imaging of membranes

TEM tomography provides the best resolution $(<1 \mathrm{~nm})$ and is therefore the best choice if fine structures need to be observed. We have used TEM tomography before to image block copolymer membranes ${ }^{22}$, which offered precise and detailed information on the membrane selective layer. TEM tomography was recently used by Pacheco et al. ${ }^{23}$ to visualize the structure of the top selective layer of thin film composite membranes. Maijoinen et al. ${ }^{24}$ and Kaushik et al. ${ }^{25}$ used 3D cryo-TEM tomography for investigating cellulose nanocrystals close to native conditions. The disadvantage of TEM tomography is the small sampling volume (ca. 2-5 micrometer in the three dimensions), which does not give an overview of the whole membrane structure. Slices thinner of $100 \mathrm{~nm}$, obtained by ultramicrotomy or focused ion beam are normally required. Additionally to the morphology normally revealed by TEM using elastic electrons, more information relative to contrast promoted by differences in crystallinity or composition can be obtained in combination with spectroscopy techniques. Examples useful technologies for this purpose are dark-field TEM, electron energy loss spectroscopy (EELS) and energy dispersive spectroscopy (EDS). ${ }^{26}$ 
Atomic Force Microscopy (AFM) provides images with high resolution with 3D visualization with relatively easy sample preparation and the advantage of observing samples at environmental conditions without need for high vacuum. Valuable achievements on quantified morphological characterization of membranes have been recently reported using AFM. ElHadidy et al. ${ }^{27,} 28$ and AlMarzooqi et al. ${ }^{28}$ worked on pore analysis for ultrafiltration membranes based on AFM images correlating with porometry techniques. The main disadvantage of AFM for 3D imaging is that it just samples the superficial layers of a membrane. The information is closer to that obtained by $2 \mathrm{D}$ imaging methods than to that supplied by other 3D characterization methods discussed here.

In opposite to TEM tomography or AFM, X-ray computed tomography ${ }^{29}$ or synchrotron radiation computed microtomography ${ }^{30,31}$ image relatively large volumes, being considered a multi-length scale methodology, which can be non-destructive and used for in situ experiments to follow process evolution (4D investigation). 3D reconstruction of polymeric membranes by X-ray tomography is being used for 3D reconstruction of polymeric ${ }^{30}$ and inorganic ${ }^{32}$ membranes. The image resolution is commonly in the micrometer range. This technology is constantly improving with the implementation of better X-ray optics in synchrotron facilities, allowing the resolution to go far bellow 1 micrometer. As far as membrane is concerned the methodology has been successful for microfiltration and solid oxide fuel cell. While the absorption contrast of X-ray tomography is more suitable for imaging ceramic and metallic structures, phase contrast X-ray imaging is becoming widespread and more successfully for low atomic number materials. A recent example is the characterization of graphite electrodes in Li-ion batteries. ${ }^{33}$

Confocal microscopy gives information on porosity in different layer depths for a porous 
structure. Snyder et al. ${ }^{34}$ reported a quantitative analysis of zeolite membranes imaged by laser scanning confocal microscopy. Confocal microscopy is a method of much more widespread and of better access to most laboratories than X-ray tomography. But the resolution of confocal is also not high, with restrictions known for optical microscopy and requires fluorescent samples. The resolution is not enough for membranes with pore size in the range of nano- or ultrafiltration. 3D reconstruction associated to the electron microscopy resolution would be more suitable.

Focused ion beam (FIB/SEM) and serial block face SEM (SBF/SEM) fill the gap between TEM and X-ray tomography (or confocal microscopy) in terms of resolution and imaged volume. FIB removes thin slices of a sample by incidence of ion beam, consecutively exposing a fresh cross section, which is imaged by the electron beam of a SEM. FIB has been much more applied to inorganic porous materials. Holzer et al. ${ }^{35,36}$ applied focused ion beam (FIB/SEM) for 3D reconstruction of porous ceramics. FIB application to polymeric membranes has been much less explored, since additional care and control of experimental parameters are required to avoid sample damage for instance when exposed to Gallium ions and charging. We were the first to report the used of FIB coupled to FESEM for a qualitative 3D-reconstruction of polymeric porous membranes ${ }^{11}$. The 3D visualization and semiautomated segmentation were carried out using software tools implemented in the program Amira (TGS, San Diego, CA). Zils et al. ${ }^{37}$ applied FIB nanotomography in a similar way for characterization of polymer electrolyte fuel cell. The alignment of the image stacks in this case was done with the software ImageJ and StackReg Plugin and further analysis by using Otsu algorithm.

Reingruber et al. ${ }^{38}$ recently published the quantitative analysis of a 3D-reconstruction of 
microfiltration membranes using SBF/SEM. The method consists of serial sectioning provided by an ultramicrotome in the specimen chamber of an ESEM, which is a variable pressure FESEM. The ESEM resolution is normally lower than that of a high vacuum FESEM, but the method was appropriate for microfiltration membranes.

In addition to Figure 1, Table 1 summarize characteristics of different 3D imaging methods, which could be applied for membranes.

Table 1. Comparison of different 3D imaging methods

\begin{tabular}{llll}
\hline Method & Image & Staining & Slicing \\
\hline TEM tomography & $\begin{array}{l}\text { Elastic electrons } \\
\text { (Inelastic electrons for } \\
\text { element contrast) }\end{array}$ & Needed & $\begin{array}{l}\text { Ultramicrotomy or ion } \\
\text { beam }\end{array}$ \\
AFM & $\begin{array}{l}\text { Probe-sample force or } \\
\text { interaction }\end{array}$ & Not required & Not required \\
FIB/SEM & $\begin{array}{l}\text { Secondary electrons } \\
\text { (backscattered } \\
\text { electrons for } \\
\text { composition contrast) }\end{array}$ & Not needed & lon beam \\
& $\begin{array}{l}\text { Backscattered } \\
\text { electrons }\end{array}$ & Needed & Ultramicrotomy \\
SBF/SEM & X-rays & $\begin{array}{l}\text { Heavy elements have } \\
\text { b-Ray Computed }\end{array}$ & Not required \\
Tomography & Light/Fluorescence & $\begin{array}{l}\text { Needed if sample not } \\
\text { fluorescent }\end{array}$ & Not required \\
Confocal microscopy & &
\end{tabular}

More than an optical visualization method, a quantitative analysis is required to fully correlate morphology to transport, selectivity and additional parameters such as concentration polarization. While 2D microscopy images do not allow measurements of 
volume specific surface area with pore length distribution and connectivity across the membrane, a 3D quantitative analysis is ideal for this purpose. We recently demonstrate how 3D image simulation can help in predicting transport characteristics in membranes ${ }^{39}$. Quantitative analysis can be incorporated as starting point for flux simulations to design better membranes. By providing experimental 3D quantitative visualization of membrane morphology, transport simulations would be even more effective. There is a need for more complete approaches, offering quantitative characterization associated to 3D reconstruction with high resolution.

Since most membranes have a gradient porosity, it would be very useful to have a quantitative analysis of pore size distribution layer by layer for a porous membrane, obtaining a complete 3D mapping of the structure. This can be hardly done experimentally. Confocal microscopy reveals the pore structure in different depths, but as mentioned above the resolution is not enough for most ultra- and nanofiltration membranes.

In this work we explore and compare two electron microscopy methods for 3D imaging, FIB/SEM and SBF/SEM, for the characterization of block copolymer membranes with pore size of 20-50 nm, a range, which requires higher resolution than for microfiltration and can not be achieved by X-ray computed tomography. We propose a new segmentation method for quantitative analysis of porosity of membranes slice sets applied to the two methods. Information on porosity can be obtained in different layers or depths. We demonstrate the feasibility, by applying the methods to membranes prepared by block copolymer selfassembly and non-solvent induced phase separation. The membranes are asymmetric with a top layer containing pores in the range of $20-50 \mathrm{~nm}$. As the depth increases, the pore size increases and the pores become less regular. The same method can be extended in principle 
to any other polymeric porous membrane.

\section{Experimental}

\section{Materials}

Polystyrene- $b$-poly(4-vinylpyridine) block copolymer (PS-b-P4VP 175000-b-65000)) (P9828-S4VP was purchased from Polymer Source, Inc., Canada. 1,4-Dioxane, dimethylformamide (DMF), gold (III) chloride and sodium citrate were supplied by SigmaAldrich. Tetrahydrofuran was supplied by Fischer Scientific. Methyl iodide used for staining was supplied by Acros-organics.

\section{Membrane preparation}

The membranes analyzed in this work were prepared by block copolymer selfassembly and non-solvent induced phase separation, a method, which has been explored by our group in the last few years. The membranes characterized by FIB/SEM and by SBF/SEM were prepared by slightly different procedures:

Procedure 1: The membrane imaged by FIB/SEM was prepared by casting a PS- $b$-P4VP solution in a mixture of dimethyl formamide and tetrahydrofuran with copper acetate, as previously reported in Ref. ${ }^{11}$.

Procedure 2: The membrane analyzed by SBF/SEM was prepared by casting a PS- $b$-P4VP solution in a 1:1:1 (wt \%) mixture of DMF, THF and dioxane, followed by immersion in water, analogous to the description reported in Ref. ${ }^{19}$. The prepared PS- $b$-P4VP membrane was then used to filter a $1 \mathrm{wt} \%$ gold (III) chloride solution. Gold ions complex with pyridine 
blocks ${ }^{40}$. The complexed gold was then reduced to gold nanoparticles by treating the membrane with a $0.05 \mathrm{M}$ sodium citrate solution.

\section{Imaging}

Two methods were used for imaging: (1) FIB/SEM and (2) SBF/SEM.

\section{Focused Ion Beam / Field Emission Scanning Electron Microscopy (FIB/SEM)}

Membranes prepared by procedure 1 were sectioned, by using focused ion beam. To minimize the polymer sample damage by the ion beam, the beam currents were lower than usually applied for semiconductors analysis, and a protective Pt layer was deposited, prior to milling the surface at the region of interest. The layer was deposited using ion beam induced CVD, inside the FIB/SEM chamber. In order to effectively dissipate surface charge from the surface, the membrane surface was also sputter- coated with Au prior to imaging/milling. The series of 150 slices (each with $50 \mathrm{~nm}$ thickness) was obtained by using a FEI Helios 400S small dual beam system and Slice \& View software.

\section{Serial Block Face Scanning Electron Microscopy (SBF/SEM)}

The morphology of membranes prepared by procedure 2 was analyzed by serial block-face scanning electron microcopy (SBF/SEM), performed with a GATAN 3View sectioning system. SBF/SEM combines environmental scanning electron microscopy and an automated ultramicrotome placed inside the microscope chamber. Backscattered electrons detector is normally used for imaging. To increase the conductivity and coefficient of backscattered electron signals, the membrane was stained with vapors of methyl iodide for 4 $\mathrm{h}$ before embedding it in resin blocks. The resin block with embedded membrane was cut off with jewelry saw and glued with epoxy resin to aluminum rivet in such a way that a profile of 
the membrane cross section would be observed once the block face is trimmed with razor blade and ultra-microtome. To minimize charging, the lateral sides of the block face were coated with colloidal silver paint and the block face was coated with $5 \mathrm{~nm}$ gold inside a sputter coater. A Quanta 200 FEG SEM (FEI) equipped with the GATAN 3View door and backscattered electron detector was used for serial sectioning and imaging of the block face. The image stack of $50 \mathrm{~nm}$ slices was acquired in an automated fashion in low vacuum mode under the following conditions: $2.0 \mathrm{kV}$ voltage, current of 43 pico Amp, resolution 1024x1024, pixel size $8 \mathrm{~nm}$, pixel time $32 \mu$ seconds, chamber pressure of 0.2239 Torr, magnification of $16,477 \mathrm{x}$. A set of 1000 slices was obtained.

\section{Segmentation method}

$3 \mathrm{D}$ reconstruction with detailed and quantitative information on pore size and porosity in different layers of the membrane requires first the segmentation of the microscopy slices images. Details of the segmentation method using the Mumford-Shah Model ${ }^{41-44}$ and an algorithm developed at KAUST for this purpose are described in the supporting information. For segmentation, the image of the membrane consists of empty spaces (pores) and membrane matrix, which have to be automatically identified and separated from each other. The image intensity in different regions is spatially homogeneous. The membrane can be considered as consisting of several regions whose image intensity is described by smooth function plus additive noise. The goal of the segmentation algorithm is to determine the regions, in which the image intensities are smooth functions. Since both the functions and regions are initially unknown, the algorithm below estimates both simultaneously. The problem described is a classical problem in computer vision, which is 
normally referred as Mumford-Shah Model ${ }^{41-44}$. Several numerical optimization methods have been proposed to solve this problem ${ }^{45,46}$, but the algorithms tend to be computationally expensive, prone to local optima, and extremely sensitive to initialization. Therefore, we employ a recent technique ${ }^{42}$, which is computationally efficient (compared to ${ }^{45}$, 46 ), less susceptible to local minima, insensitive to initialization, and therefore, may be employed in a fully automated scheme.

\section{Results and Discussion}

The first method explored in this work was FIB/SEM. FIB has been recognized as a successful method for morphology investigation of different kinds of materials, but has been particularly well applied for nanofabrication in the semi conductor industry ${ }^{47}$ or preparation of ceramic samples for transmission microscopy ${ }^{48}$, which could not be easily cut by ultramicrotomy. More recently FIB/SEM has been increasingly used for the characterization of biological samples ${ }^{49}$. The use of FIB for polymeric membranes has been much more restrict and a reason for that is the potential damage of the sample by exposure to the beam, if the conditions are not properly controlled. Boussu et al. ${ }^{50}$ were one of the first to report the use of FIB for polymeric membrane characterization. However they used it only to expose cross-sections of different polymeric membranes for SEM investigation in an alternative way to the classical sample fracture. Our group was probably the pioneer in using FIB sectioning for $3 \mathrm{D}$ reconstruction of polymeric porous membranes, as reported 6 years ago ${ }^{11}$. Low beam currents and a protective Pt layer were applied to minimize damages. Our previous report used FIB for qualitative visualization purpose. Here we propose a method to quantify porosity in different membrane layers, demonstrating it for asymmetric porous membranes. Typical membrane slices 2D images obtained by FIB/SEM are shown in Figure 2. 

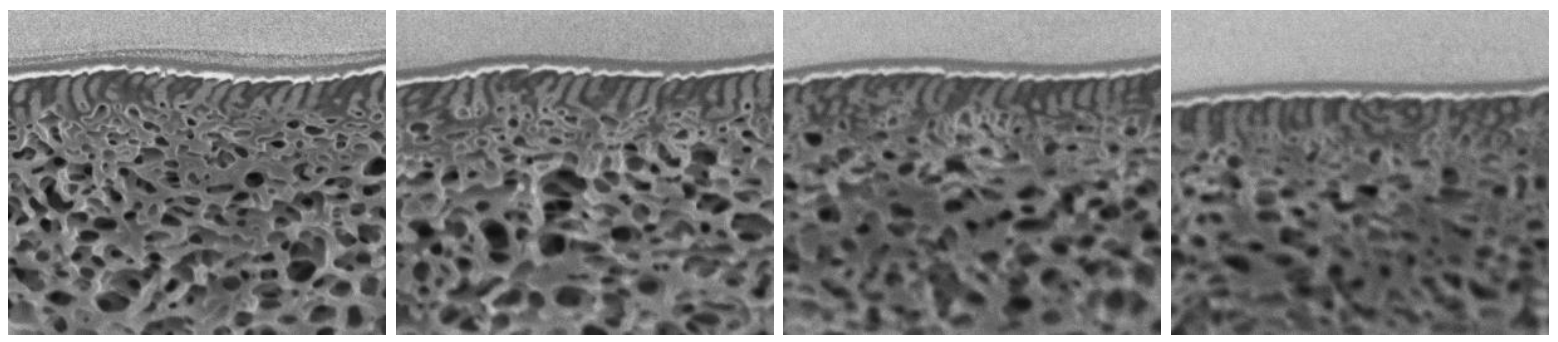

$500 \mathrm{~nm}$

Figure 2. FIB/SEM Membrane slices images to be segmented.

Figure 3 shows the 3D reconstruction of the membrane from slices obtained by FIB/SEM, similar to those depicted in Figure 2. Figure $3 \mathrm{~b}$ represents the first step of segmentation.

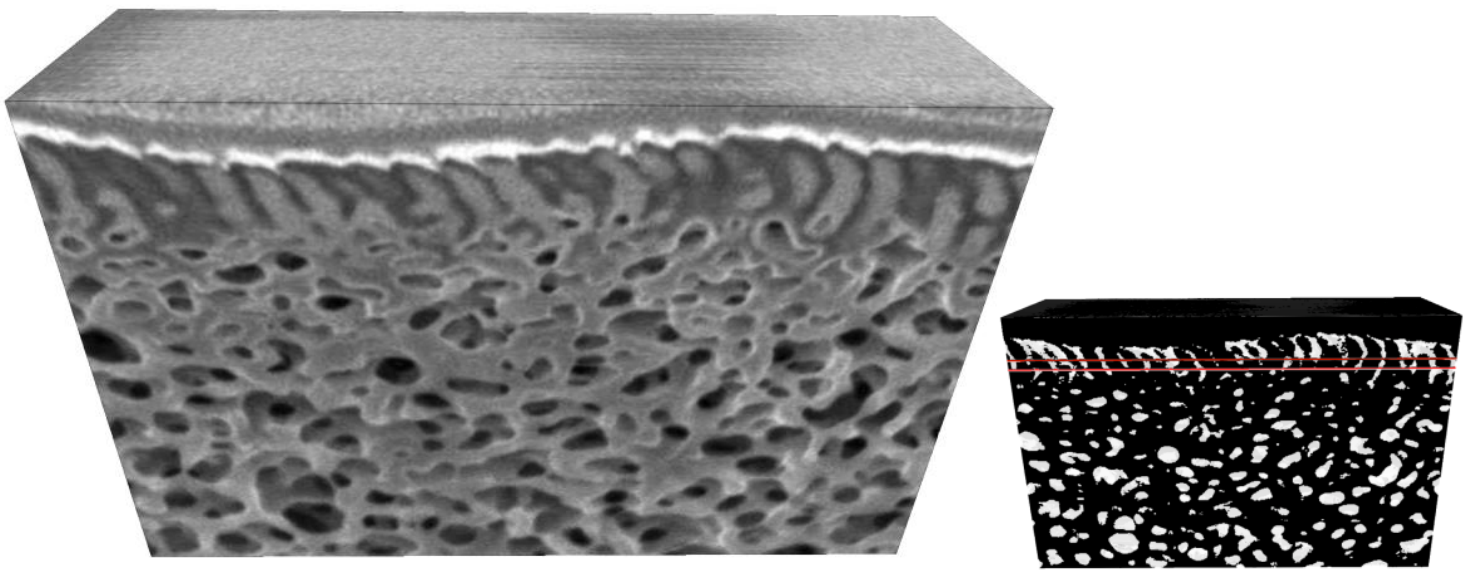

(a)

(b)

Figure 3. (a) 3D visualization of the membrane reconstructed from the set of slices obtained by FIB/SEM and respective (b) segmentation.

FIB/SEM allows the imaging of slices obtained orthogonal to the membrane surface. For practical reasons, slices parallel to the membrane surface can be hardly experimentally obtained. By 3D reconstruction and application of the developed algorithm, the porosity and pore average size in different layers parallel to the membrane surface can be now quantified, giving a complete picture of the membrane. 


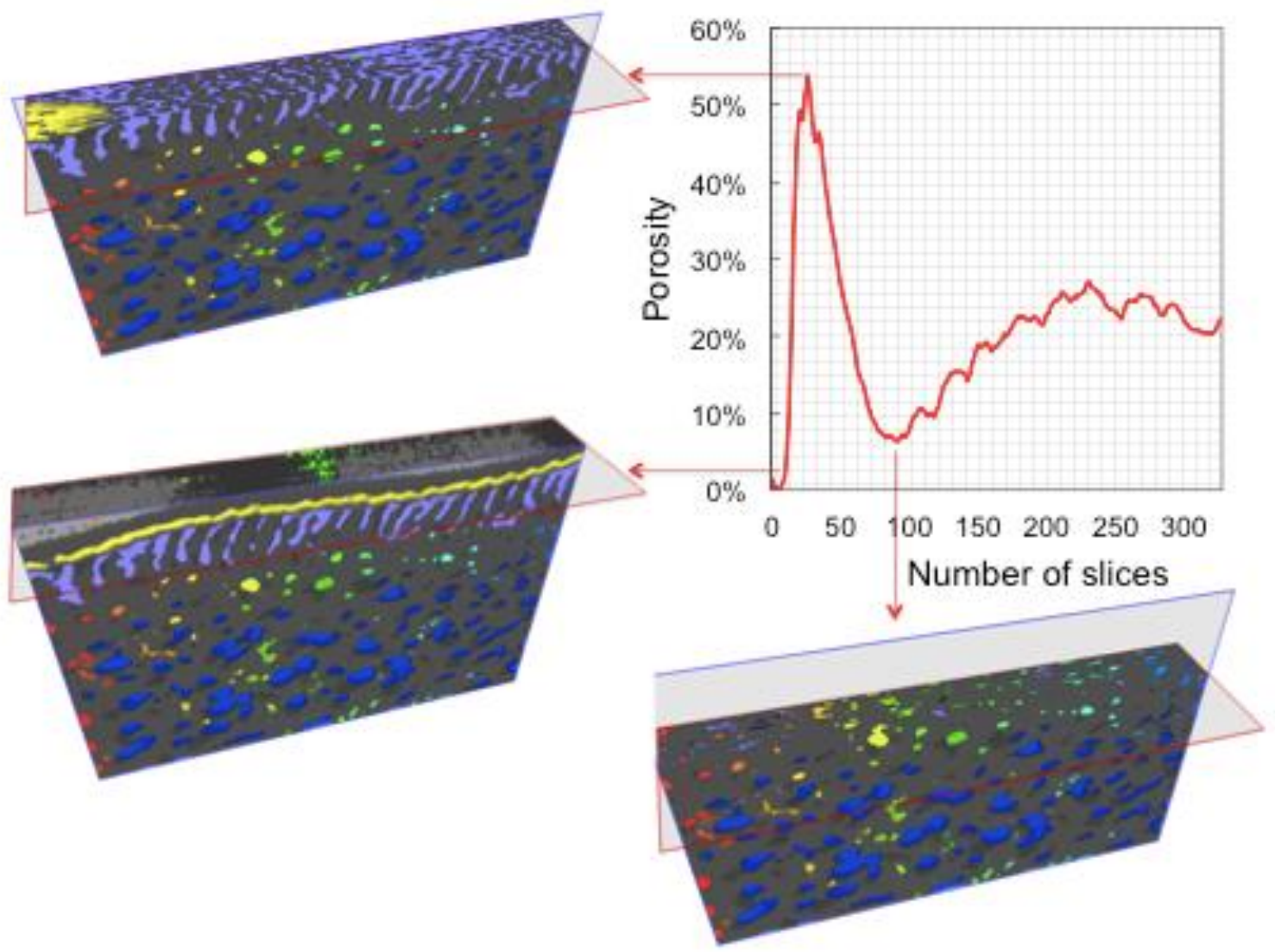

Figure 4. Porosity layer by layer, calculated for each virtual slice parallel to the membrane surface, each slice with thickness of $50 \mathrm{~nm}$. Images obtained by FIB/SEM.

Figure 4 shows the results of the porosity calculation using the algorithm, as well as the visualization of different cuts corresponding to layers of different porosity. 


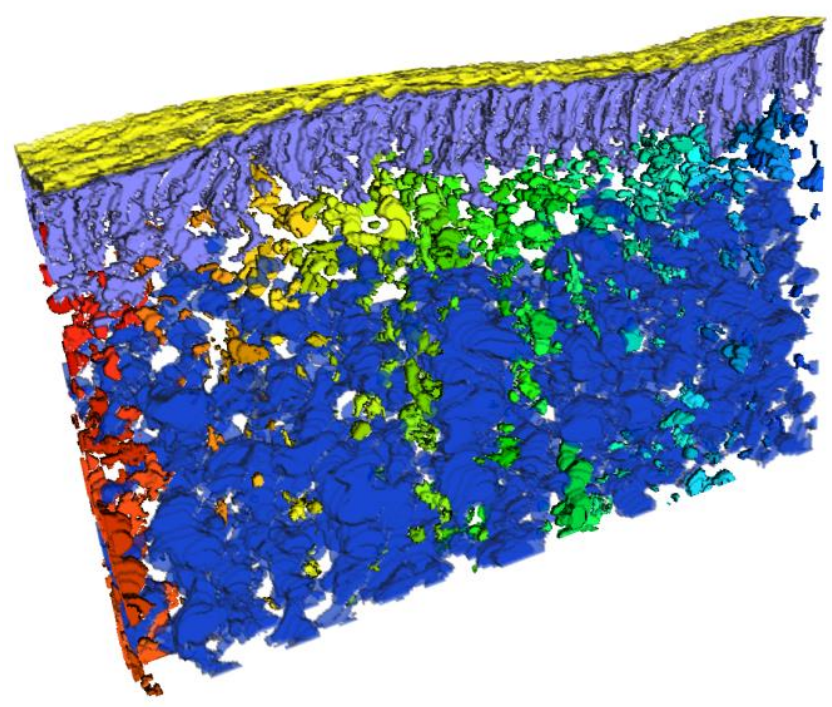

Figure 5. 3D-Reconstruction of the membrane, after segmentation and algorithm application. Each color represents pore regions with interconnectivity.

As a result of the segmentation it is possible also to distinguish the pore regions, which are highly interconnected. This is visualized in Figure 5, with different colors representing regions with preferential inter-pore connectivity. 


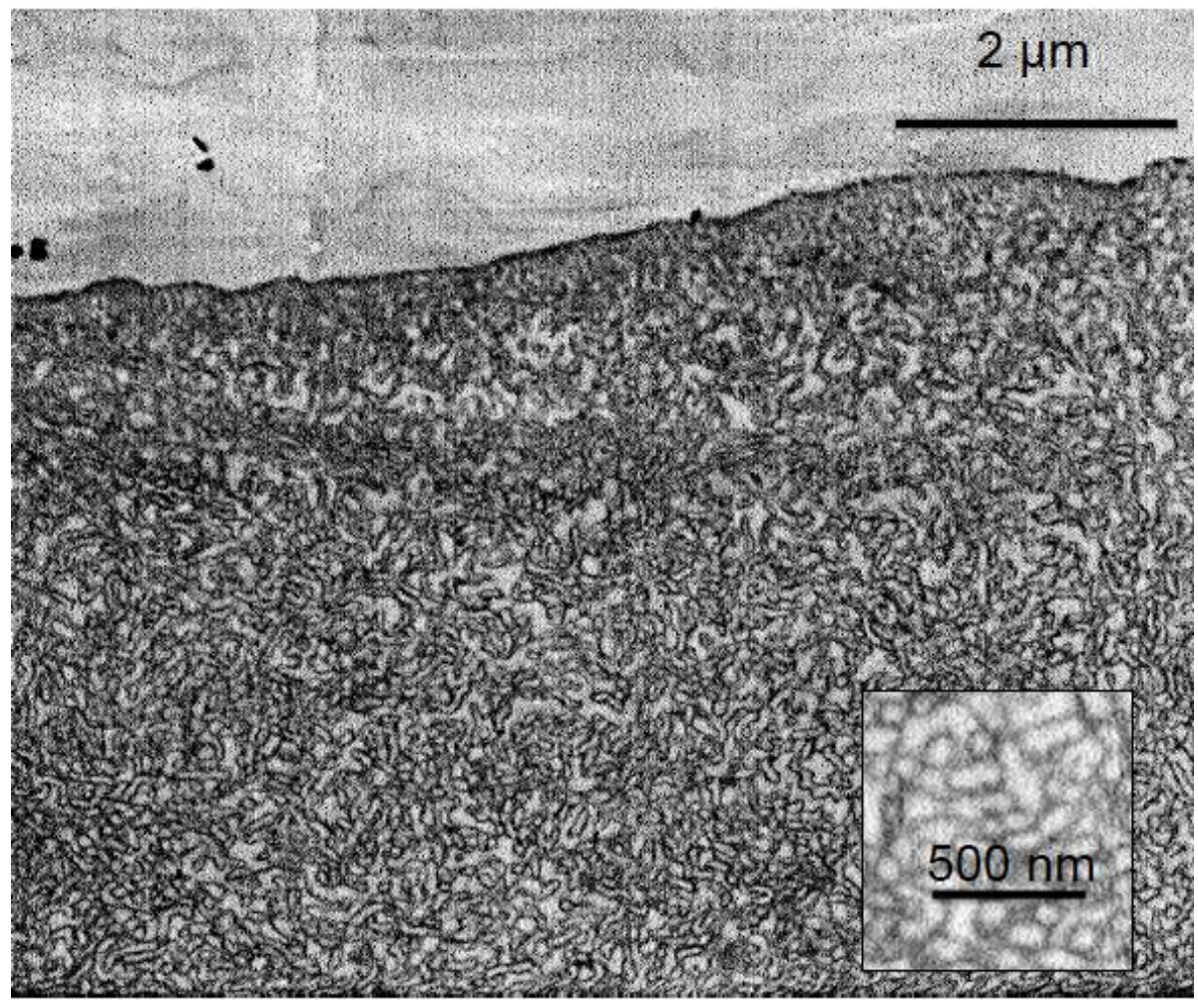

Figure 6. Membrane slice, obtained by SBF/SEM, after staining with $\mathrm{CH}_{3} \mathrm{I}$ and gold.

The second imaging method used in this work was SBF/SEM. We used for that a GATAN 3View system. A typical slice image can be seen in Figure 6. The sample for FIB/SEM was not embedded in resin and the contrast for each slice was topographic, using secondary electrons, analogously to the images obtained by regular FESEM. For SBF/SEM the sample was embedded in resin and sliced by ultramicroty inside the microscope chamber. Since the imaged surface is smooth, the contrast is provided by differences in local composition. The image is formed by backscattered electrons. The contrast given by atoms constituting each blocks of a plain membrane and the resin is not enough for a satisfactory image. SBF/SEM is convenient to image structures already with heavy elements such as samples decorated with inorganic nanoparticles. If the image of plain polymeric membranes is the target, additional staining is necessary. For biological or synthetic polymers, staining 
with osmium tetroxide is a common procedure. The block copolymer we used contains styrene and pyridine blocks. Pyridine can be selectively stained by $\mathrm{CH}_{3} \mathrm{I}$. Pyridine complexes with gold ions, which provide even stronger contrast. The membrane is preformed and then stained by exposure to the vapor phase $\left(\mathrm{CH}_{3} \mathrm{I}\right)$ or by filtration of an aqueous gold solution, followed by reduction to elementary gold. Under these conditions the membrane morphology is not expected to change, but the it must be clear that the dark regions in Figure 6 corresponds to regions occupied mainly by pyridine blocks. For the membranes investigated here by SBF/SEM, gold was not only added for pure staining purposes, but have been also previously explored for catalytic purpose. ${ }^{40}$

As for the FIB/SEM slices, segmentation and 3D reconstruction were conducted for SBF/SEM images and the porosity was quantitatively analyzed and presented in Figure 7. As in Figure 5, different colors emphasize pore regions with high interconnectivity. 


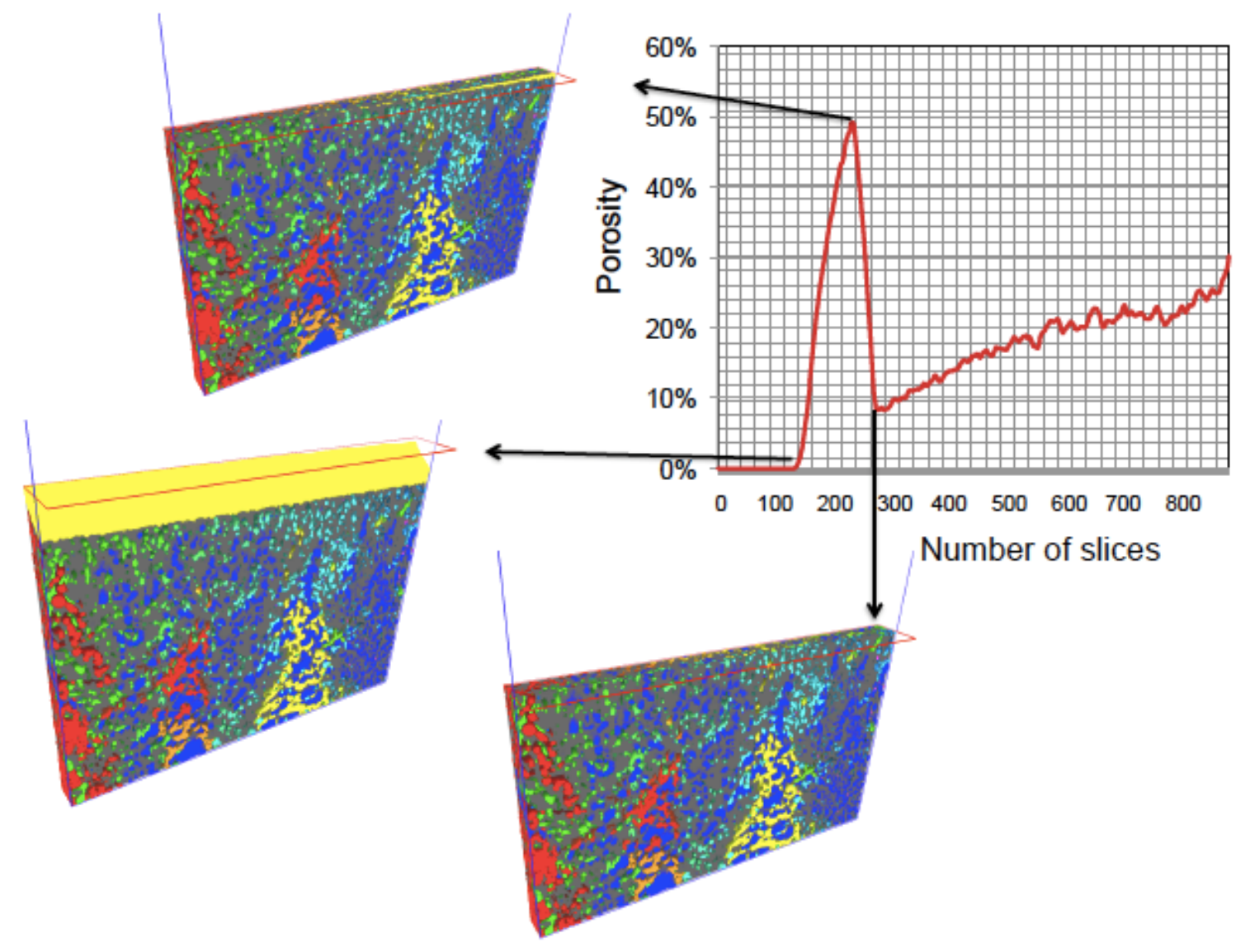

Figure 7. Porosity layer by layer, calculated for each virtual slice parallel to the membrane surface, each slice with thickness of $50 \mathrm{~nm}$. The images were obtained by SBT/SEM, by using a GATAN 3View.

Both imaging methodologies used in this work gave similar quantitative information on porosity. The convenience of using one or other depends on the characteristic of membrane being characterized, contrast for backscattering electrons and stability in the ion beam. Figure 6 for SBF/SEM is closer to images obtained by TEM and takes advantage of analogous staining or intrinsic contrast provided by elements of higher electron density or different capacity of scattering electrons. A diamond knife is used as for TEM. The samples 
must be sliceable by the knife and properly prepared, including adequate embedding in resin. SBF/SEM can in principle sample larger volumes in shorter times than FIB/SEM, as claimed also for other materials ${ }^{49,51}$. While for the membranes investigated in this work SBF/SEM was a very convenient method, the same staining might not work for membranes based on other materials, which do not complex with gold or do not strongly interact with $\mathrm{CH}_{3} \mathrm{I}$. Other staining methods need then to be evaluated. Membranes containing acrylic acid blocks can easily complex with other metal ions. For membranes without charged segments long staining with osmium tetroxide before embedding might provide enough contrast for SBF/SEM. According to previous literature ${ }^{49}$ for instance for biological samples, whose characteristics are not much different than that of polymeric membranes, FIB/SEM is slightly superior in resolution and lower in terms of coverable volumes. By using backscattered electrons instead of secondary electrons for FIB/SEM, it would be possible to obtain additional composition contrast, however with lower image resolution. In general both SBF/SEM and FIB/SEM technologies are developing fast with improvement of resolution. FIB/SEM would be the method of choice in the case of ceramic membranes or other hard materials, which should not be cut by diamond knifes. Both SBF/SEM and FIB/SEM cover much larger volumes than TEM tomography. We have used TEM tomography before ${ }^{52}$ for membrane characterization, which provides excellent resolution, but the imaged area is restricted. The possibility of imaging a large part of the membrane, typically with thickness of 20-50 micrometer or more, can be very useful to have a complete understanding of transport. Both SBF/SEM and FIB/SEM cover this range. The membranes investigated here have pores larger than $20 \mathrm{~nm}$ and for that the resolution of both methods is sufficient. This would be the case for most ultrafiltration membranes. When imaging smaller pores, the 
resolution of FIB/SEM could be advantageous.

The results obtained by applying segmentation, 3D reconstruction and the developed algorithm allowed the quantification of porosity in any layer parallel to the surface going from one side to the other of the membrane. Also information on the pore interconnectivity in adjacent areas is provided. This is very useful in the field of synthetic membranes. Most commercial membranes for ultra- nanofiltration and reverse osmosis as well as gas separation are asymmetric membranes with complex morphology. Morphological characterization is essential as tool for membrane development and to take decisions on best choices for each application and process optimization. For fully understanding or simulating transport in membranes the $3 \mathrm{D}$ reconstruction and analysis described in this paper can be much more useful than regular 2D images obtained by SEM or TEM, giving the complete view and quantitative information. Here we provide experimental 3D images and we anticipate that this information could be used in combination with transport simulation tools similar to those used in Ref. ${ }^{39}$ and additional methods under development for a much better transport and structure optimization in the near future.

\section{Conclusion}

Two microscopy methods for 3D imaging visualization, FIB/SEM and SBF/SEM, were applied to porous block copolymer membranes. Both methods offered high resolution for adequate pore analysis and covered sample volumes higher than normally possible by transmission electron microscopy tomography. With help of a new algorithm, the set of images obtained by both methods could be analyzed to provide a complete mapping of

porosity and average pore size in different membrane layers. The 3D-visualization 
combining information gained by the image segmentation showed the pore connectivity in different membrane regions. We demonstrate here how effective the method is for asymmetric block copolymer membranes. The same analysis using the proposed algorithm can be applied for any kind of membranes or porous materials. The imaging method to be chosen depends however on the mechanical properties, feasibility of slicing with diamond knife or ion beam, pore size, volume to be sampled and feasibility of staining. The method is

particularly valuable for asymmetric membranes, which are used in many applications. It can help the development of new systems for forward osmosis and other applications for which not only the porosity of the top selective layer is relevant, but also that of the whole porous substrates.

\section{Supporting information}

The proposed algorithm used for segmentation and quantification of porosity in different layers is detailed in the supporting information.

\section{Acknowledgements}

This work was supported by the King Abdullah University of Science and Technology (KAUST), KAUST Project "Visualization and Pore Tuning of Asymmetric Membranes". 


\section{References}

1. Nunes, S. P.; Peinemann, K.-V., Membrane technology: in the chemical industry. John Wiley \& Sons: 2006.

2. Shannon, M. A.; Bohn, P. W.; Elimelech, M.; Georgiadis, J. G.; Mariñas, B. J.; Mayes, A. M., Science and technology for water purification in the coming decades. Nature 2008, 452, (7185), 301-310.

3. Loeb, S.; Titelman, L.; Korngold, E.; Freiman, J., Effect of porous support fabric on osmosis through a Loeb-Sourirajan type asymmetric membrane. Journal of Membrane Science 1997, 129, (2), 243-249.

4. Lee, A.; Elam, J. W.; Darling, S. B., Membrane materials for water purification: design, development, and application. Environmental Science: Water Research \& Technology 2016, 2, (1), 17-42.

5. Gruber, M.; Johnson, C.; Tang, C.; Jensen, M. H.; Yde, L.; Hélix-Nielsen, C., Computational fluid dynamics simulations of flow and concentration polarization in forward osmosis membrane systems. Journal of Membrane Science 2011, 379, (1), 488495.

6. Li, W.; Gao, Y.; Tang, C. Y., Network modeling for studying the effect of support structure on internal concentration polarization during forward osmosis: model development and theoretical analysis with FEM. Journal of Membrane Science 2011, 379, (1), 307-321.

7. Park, M.; Lee, J. J.; Lee, S.; Kim, J. H., Determination of a constant membrane structure parameter in forward osmosis processes. Journal of Membrane Science 2011, 375, (1), 241-248.

8. $\quad$ Maab, H.; Al Saadi, A.; Francis, L.; Livazovic, S.; Ghafour, N.; Amy, G. L.; Nunes, S. P., Polyazole hollow fiber membranes for direct contact membrane distillation. Industrial \& Engineering Chemistry Research 2013, 52, (31), 10425-10429.

9. $\quad$ Prehn, K.; Adelung, R.; Heinen, M.; Nunes, S. P.; Schulte, K., Catalytically active CNT-polymer-membrane assemblies: From synthesis to application. Journal of Membrane Science 2008, 321, (1), 123-130.

10. Tripathi, B. P.; Schieda, M.; Shahi, V. K.; Nunes, S. P., Nanostructured membranes and electrodes with sulfonic acid functionalized carbon nanotubes. Journal of Power Sources 2011, 196, (3), 911-919.

11. Nunes, S. P.; Sougrat, R.; Hooghan, B.; Anjum, D. H.; Behzad, A. R.; Zhao, L.; Pradeep, N.; Pinnau, I.; Vainio, U.; Peinemann, K.-V., Ultraporous films with uniform nanochannels by block copolymer micelles assembly. Macromolecules 2010, 43, (19), 8079-8085.

12. Nunes, S. P.; Behzad, A. R.; Hooghan, B.; Sougrat, R.; Karunakaran, M.; Pradeep, N.; Vainio, U.; Peinemann, K.-V., Switchable pH-responsive polymeric membranes prepared via block copolymer micelle assembly. ACS nano 2011, 5, (5), 3516-3522.

13. Nunes, S. P.; Karunakaran, M.; Pradeep, N.; Behzad, A. R.; Hooghan, B.; Sougrat, R.; He, H.; Peinemann, K.-V., From micelle supramolecular assemblies in selective solvents to isoporous membranes. Langmuir 2011, 27, (16), 10184-10190.

14. Jackson, E. A.; Hillmyer, M. A., Nanoporous membranes derived from block copolymers: From drug delivery to water filtration. ACS nano 2010, 4, (7), 3548-3553. 
15. Seo, M.; Moll, D.; Silvis, C.; Roy, A.; Querelle, S.; Hillmyer, M. A., Interfacial Polymerization of Reactive Block Polymers for the Preparation of Composite Ultrafiltration Membranes. Industrial \& Engineering Chemistry Research 2014, 53, (48), 18575-18579.

16. Nunes, S. P.; Car, A., From charge-mosaic to micelle self-assembly: block copolymer membranes in the last 40 years. Industrial \& Engineering Chemistry Research 2012, 52, (3), 993-1003.

17. Nakao, S.-i., Determination of pore size and pore size distribution: 3. Filtration membranes. Journal of Membrane Science 1994, 96, (1), 131-165.

18. Marques, D. S.; Dorin, R. M.; Wiesner, U.; Smilgies, D.-M.; Behzad, A. R.; Vainio, U.; Peinemann, K.-V.; Nunes, S. P., Time-resolved GISAXS and cryo-microscopy characterization of block copolymer membrane formation. Polymer 2014, 55, (6), 1327 1332.

19. Marques, D. S.; Vainio, U.; Chaparro, N. M.; Calo, V. M.; Bezahd, A. R.; Pitera, J. W.; Peinemann, K.-V.; Nunes, S. P., Self-assembly in casting solutions of block copolymer membranes. Soft Matter 2013, 9, (23), 5557-5564.

20. Mottern, M. L.; Shqau, K.; Shi, J.; Yu, D.; Verweij, H., Thin supported inorganic membranes for energy-related gas and water purification. International Journal of Hydrogen Energy 2007, 32, (16), 3713-3723.

21. Kyotani, T.; Mizuno, T.; Katakura, Y.; Kakui, S.; Shimotsuma, N.; Saito, J.; Nakane, $\mathrm{T}$., Characterization of tubular zeolite $\mathrm{NaA}$ membranes prepared from clear solutions by FTIR-ATR, GIXRD and FIB-TEM-SEM. Journal of membrane science 2007, 296, (1), 162170.

22. Madhavan, P.; Sougrat, R.; Behzad, A. R.; Peinemann, K.-V.; Nunes, S. P., Ionic liquids as self-assembly guide for the formation of nanostructured block copolymer membranes. Journal of Membrane Science 2015, 492, 568-577.

23. Pacheco, F.; Sougrat, R.; Reinhard, M.; Leckie, J. O.; Pinnau, I., 3D visualization of the internal nanostructure of polyamide thin films in RO membranes. Journal of Membrane Science 2016, 501, 33-44.

24. Majoinen, J.; Haataja, J. S.; Appelhans, D.; Lederer, A.; Olszewska, A.; Seitsonen, J.; Aseyev, V.; Kontturi, E.; Rosilo, H.; Österberg, M., Supracolloidal multivalent interactions and wrapping of dendronized glycopolymers on native cellulose nanocrystals. Journal of the American Chemical Society 2014, 136, (3), 866-869.

25. Kaushik, M.; Basu, K.; Benoit, C.; Cirtiu, C. M.; Vali, H.; Moores, A., Cellulose Nanocrystals as Chiral Inducers: Enantioselective Catalysis and Transmission Electron Microscopy 3D Characterization. Journal of the American Chemical Society 2015, 137, (19), 6124-6127.

26. Schryvers, D.; Cao, S.; Tirry, W.; Idrissi, H.; Van Aert, S., Advanced threedimensional electron microscopy techniques in the quest for better structural and functional materials. Science and Technology of Advanced Materials 2016.

27. ElHadidy, A. M.; Peldszus, S.; Van Dyke, M. I., Development of a pore construction data analysis technique for investigating pore size distribution of ultrafiltration membranes by atomic force microscopy. Journal of Membrane Science 2013, 429, 373383. 
28. AlMarzooqi, F. A.; Bilad, M.; Mansoor, B.; Arafat, H. A., A comparative study of image analysis and porometry techniques for characterization of porous membranes. Journal of Materials Science 2016, 51, (4), 2017-2032.

29. Dai, S.; Seol, Y.; Wickramanayake, S.; Hopkinson, D., Characterization of hollow fiber supported Ionic liquid membranes using microfocus X-ray computed tomography. Journal of Membrane Science 2015, 492, 497-504.

30. Remigy, J.-C.; Meireles, M.; Thibault, X., Morphological characterization of a polymeric microfiltration membrane by synchrotron radiation computed microtomography. Journal of Membrane Science 2007, 305, (1), 27-35.

31. Tung, K.-L.; Chang, K.-S.; Wu, T.-T.; Lin, N.-J.; Lee, K.-R.; Lai, J.-Y., Recent advances in the characterization of membrane morphology. Current Opinion in Chemical Engineering 2014, 4, 121-127.

32. Shearing, P. R.; Eastwood, D. S.; Bradley, R. S.; Gelb, J.; Cooper, S. J.; Tariq, F.; Lee, P., Exploring electrochemical devices using X-ray microscopy: 3D micro-structure of batteries and fuel cells. Microsc. Anal 2013, 27, (2), 19-22.

33. Eastwood, D.; Bradley, R.; Tariq, F.; Cooper, S.; Taiwo, O.; Gelb, J.; Merkle, A.; Brett, D.; Brandon, N.; Withers, P., The application of phase contrast X-ray techniques for imaging Li-ion battery electrodes. Nuclear Instruments and Methods in Physics Research Section B: Beam Interactions with Materials and Atoms 2014, 324, 118-123.

34. Snyder, M.; Vlachos, D.; Nikolakis, V., Quantitative analysis of membrane morphology, microstructure, and polycrystallinity via laser scanning confocal microscopy: application to NaX zeolite membranes. Journal of Membrane Science 2007, 290, (1), 1-18.

35. Holzer, L.; Indutnyi, F.; Gasser, P.; Münch, B.; Wegmann, M., Three - dimensional analysis of porous BaTiO3 ceramics using FIB nanotomography. Journal of Microscopy 2004, 216, (1), 84-95.

36. Mangipudi, K.; Radisch, V.; Holzer, L.; Volkert, C., A FIB-nanotomography method for accurate 3D reconstruction of open nanoporous structures. Ultramicroscopy 2016.

37. Zils, S.; Timpel, M.; Arlt, T.; Wolz, A.; Manke, I.; Roth, C., 3D visualization of PEMFC electrode structures using FIB tomography. Fuel Cells 2010, 10, (6), 966.

38. Reingruber, H.; Zankel, A.; Mayrhofer, C.; Poelt, P., Quantitative characterization of microfiltration membranes by 3D reconstruction. Journal of Membrane Science 2011, 372, (1), 66-74.

39. Shi, M.; Printsypar, G.; Iliev, O.; Calo, V. M.; Amy, G. L.; Nunes, S. P., Water flow prediction for membranes using 3D simulations with detailed morphology. Journal of Membrane Science 2015, 487, 19-31.

40. Hilke, R.; Pradeep, N.; Madhavan, P.; Vainio, U.; Behzad, A. R.; Sougrat, R.; Nunes, S. P.; Peinemann, K.-V., Block copolymer hollow fiber membranes with catalytic activity and pH-response. ACS applied materials \& interfaces 2013, 5, (15), 7001-7006.

41. Blake, A.; Zisserman, A., Visual reconstruction. MIT press Cambridge: 1987; Vol. 2. 42. Hong, B.-W.; Lu, Z.; Sundaramoorthi, G. In A new model and simple algorithms for multi-label mumford-shah problems, Computer Vision and Pattern Recognition (CVPR), 2013 IEEE Conference on, 2013; IEEE: 2013; pp 1219-1226.

43. Merriman, B.; Bence, J. K.; Osher, S., Diffusion generated motion by mean curvature. Department of Mathematics, University of California, Los Angeles: 1992. 
44. Mumford, D.; Shah, J., Optimal approximations by piecewise smooth functions and associated variational problems. Communications on pure and applied mathematics 1989, 42, (5), 577-685.

45. Tsai, A.; Yezzi Jr, A.; Willsky, A. S., Curve evolution implementation of the Mumford-Shah functional for image segmentation, denoising, interpolation, and magnification. Image Processing, IEEE Transactions on 2001, 10, (8), 1169-1186.

46. Vese, L. A.; Chan, T. F., A multiphase level set framework for image segmentation using the Mumford and Shah model. International journal of computer vision 2002, 50, (3), 271-293.

47. Cooper, D.; Rivallin, P.; Guegan, G.; Plantier, C.; Robin, E.; Guyot, F.; Constant, I., Field mapping of focused ion beam prepared semiconductor devices by off-axis and dark field electron holography. Semiconductor Science and Technology 2013, 28, (12), 125013.

48. Volkert, C. A.; Minor, A. M., Focused ion beam microscopy and micromachining. MRS bulletin 2007, 32, (05), 389-399.

49. Kizilyaprak, C.; Daraspe, J.; Humbel, B., Focused ion beam scanning electron microscopy in biology. Journal of microscopy 2014, 254, (3), 109-114.

50. Boussu, K.; De Baerdemaeker, J.; Dauwe, C.; Weber, M.; Lynn, K. G.; Depla, D.; Aldea, S.; Vankelecom, I. F.; Vandecasteele, C.; Van der Bruggen, B., Physico - Chemical Characterization of Nanofiltration Membranes. ChemPhysChem 2007, 8, (3), 370-379.

51. Müllner, T.; Zankel, A.; Svec, F.; Tallarek, U., Finite-size effects in the 3D reconstruction and morphological analysis of porous polymers. Materials Today 2014, 17, (8), 404-411.

52. Madhavan, P.; Sougrat, R.; Behzad, A. R.; Peinemann, K.-V.; Nunes, S. P., Ionic Liquids As Self-Assembly Guide for the Formation of Nanostructured Block Copolymer Membranes. Journal of Membrane Science 2015. 